the guidelines on the private sector. A bill seeking to do so, and requiring the registration of all industrial activities using recombinant DNA techniques, has been intr?duced by Senator Adla Stevenson Jr; but it has so far failed to gain any significant support, either inside Congress or without.

David Dickson

\section{Comecon Science} \section{High T, Low K}

A NEW Comecon International Laboratory of High Magnetic Fields and Low Temperatures is to be built in Wroclaw during the next Five Year Plan (1971-1985) by the Academies of Science of Poland, Bulgaria, the Soviet Union and East Germany, with possible participation also by Czechoslovakia. This will replace the existing somewhat cramped premises which have served the laboratory since 1968.

According to $\mathrm{Dr}$ Wlodzimierz Trzebiatowski, until recently the Director of the Laboratory, and who still, in retirement, takes a keen personal interest in it, Wroclaw was originally chosen as the site of the laboratory for a number of practical reasons. First the Polish Academy of Sciences already had a flourishing Institute of Low Temperature and Structure Research in Wroclaw. Wroclaw is near the important East German science centres of Dresden and Berlin, and helium, essential for low temperature work, is available from a Polish extraction plant only $100 \mathrm{~km}$ away. Finally, just as the laboratory was being planned, the Lower Sjleskan electricity board, was installing new generators in the Wroclaw tramway substation and was willing to donate the old generators to the Academy of Science.

The present laboratory is concerned with such things as the properties of hard semiconductors, new magnetic materials and the measurement of specific heats below $1^{\circ} \mathrm{K}$. Cooling below $4.2^{\circ} \mathrm{K}$ is effected by helium cooling in the field of superconducting magnets of $5 \mathrm{~T}$ and $15 \mathrm{~T}$, and work is in progress on adiabatic demagnetization of nuclear and electron paramagnetics, allowing temperatures below $0.1 \mathrm{~K}$ to be attained.

The laboratory has a special interest in compounds of uranium with non-metals of the $\mathrm{Vb}$ and VIb groups - a speciality begun by Professor Trzebiatowski at the Low Temperature Institute of the Academy of Sciences in the early 1950s. (Since his initial discovery of ferromagnetic transition in uranium hydride $\mathrm{UH}_{3}$, some fifty such uranium compounds, mostly chalcogenides, have been discovered there.) One new project, of especial interest to Dr Evgeni Leyarovski, of the Bulgarian Academy of Sciences, who was Deputy Director of the Wroclaw Laboratory from 1974-1977, is the study of superconductors

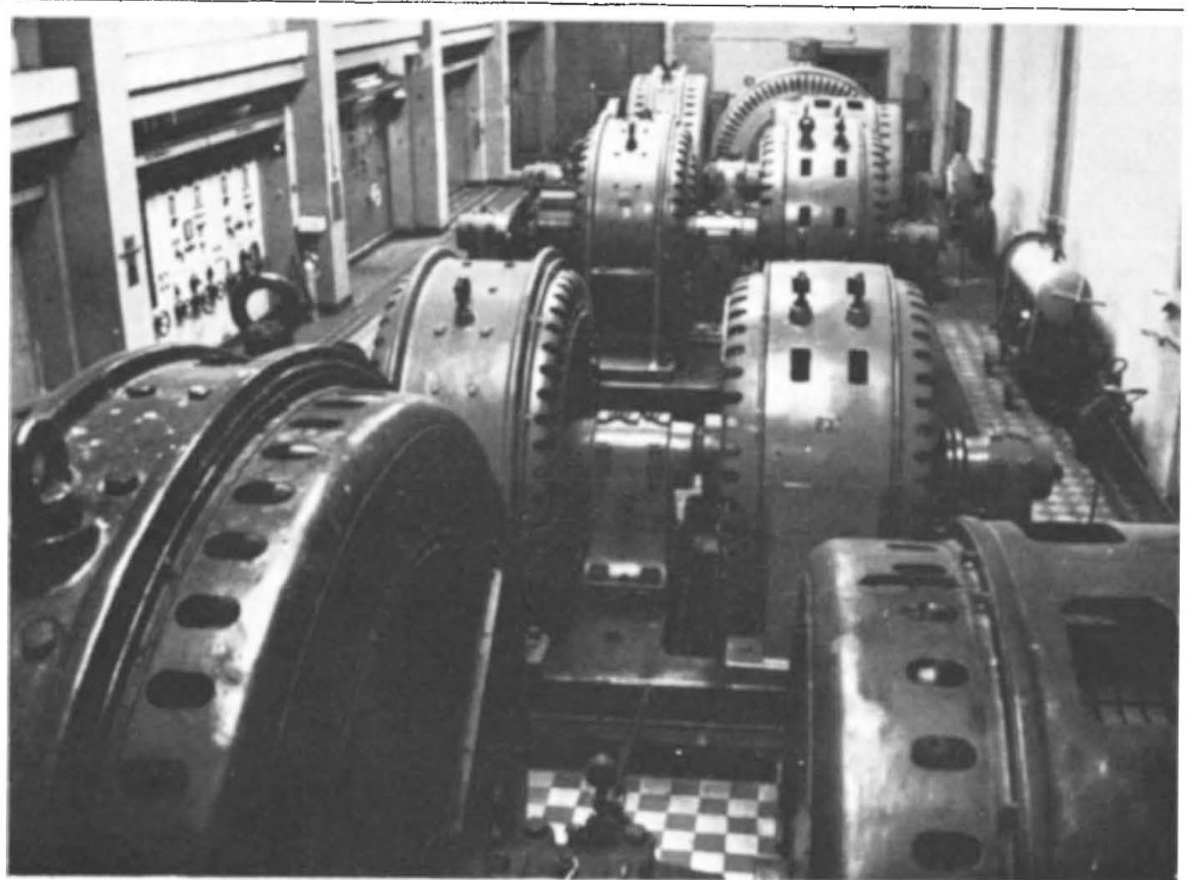

Tramway generators keep turning in Wroclaw

with magnetic impurities. Research on this phenomenon is also going forward in Sofia, but even with their new 5 tesla superconducting magnets installed this spring, the Bulgarians will still have to do much of their work in Wroclaw.

Nevertheless, even Wroclaw cannot at present offer the facilities which an international Comecon laboratory needs. Far more laboratory space is required, and, more important, the ex-tramway generators can operate only one of the three magnets at a time, so that the various teams often have to queue for "magnetic time". The new site, on the banks of the Odra, will provide space and facilities for more magnets with higher fields as well as an ample supply of water for cooling the electromagnets.

Vera Rich

\section{Soviet oil}

\section{No real shortage}

THE Soviet Union has initiated a major change in its method of estimating oil production costs which will result in a dramatic increase in production from proven fields, says a report* published last week by PetroStudies, a Swedish group specialising in the analysis of the Soviet oil and gas industry. The report disagrees sharply with CIA forecasts that the Soviet Union will suffer a shortfall in oil production. The CIA prediction has been used repeatedly in recent months by US oil officials to suggest that a Soviet oil shortage was leading it to have a material

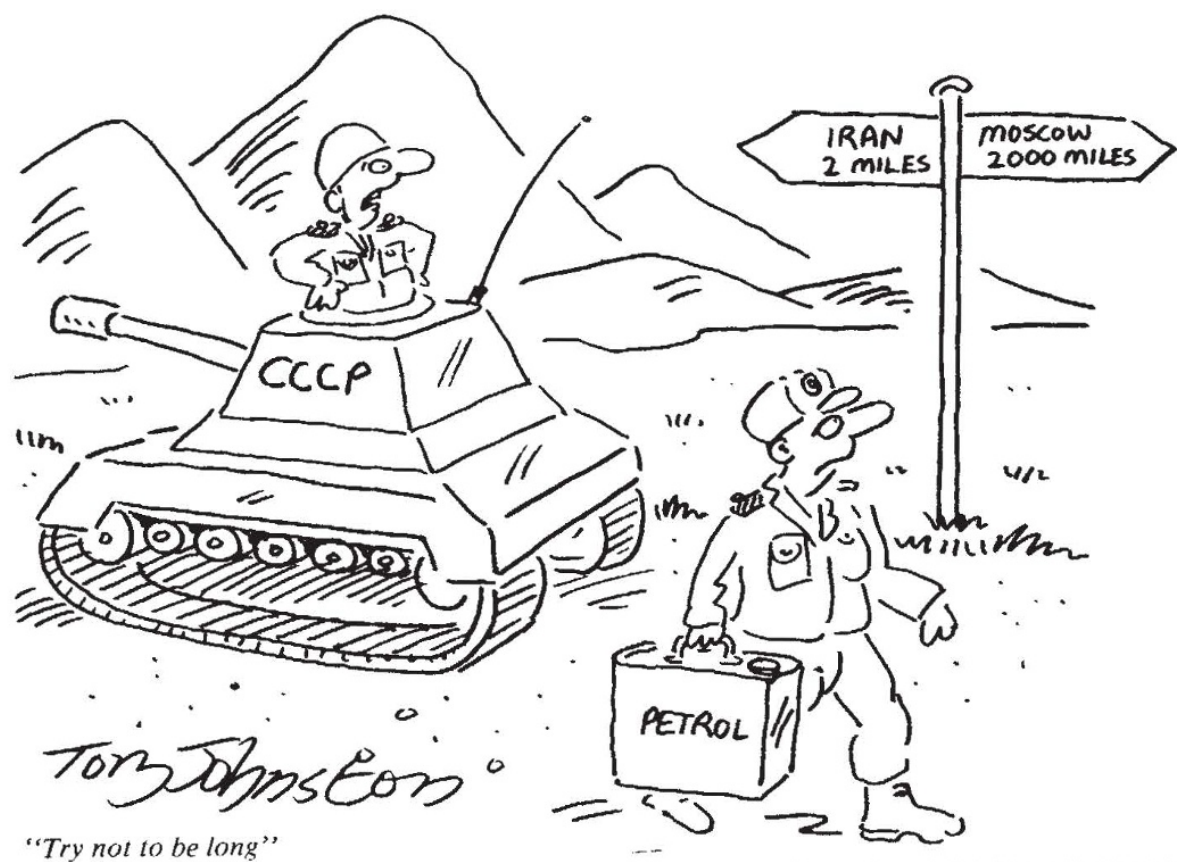

